\title{
Inhalt (detailliert)
}

\section{Band 1}

Vorwort............................. 1

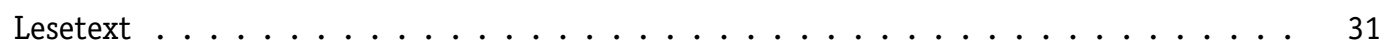

Konzeption 1: Ein Sklavenball - Butlerus . . . . . . . . . . . . . . . 33

Fragmentarische Fassung des I. Aktes $\left(\mathrm{K}^{1} / \mathrm{TS}^{1}\right) \ldots \ldots \ldots 35$

Figurenliste $\left(\mathrm{K}^{1} / \mathrm{E}^{1}\right) \ldots \ldots \ldots \ldots \ldots$

Fragmentarische Fassung des I. Aktes $\left(\mathrm{K}^{1} / \mathrm{TS}^{2}\right) \ldots \ldots \ldots$. . . . . . . . . . . . . . . . . . . . . .

Strukturplan, Figurenliste $\left(\mathrm{K}^{1} / \mathrm{E}^{2}-\mathrm{E}^{3}\right) \ldots \ldots \ldots \ldots$. . . . . . . . . . . . . . . . . . . . 44

Fragmentarische Fassung des ersten Aktes $\left(\mathrm{K}^{1} / \mathrm{TS}^{3} / \mathrm{A}^{2}\right) \ldots \ldots \ldots$. . . . . . . . . . . . . . . . .

Fragmentarische Fassung des ersten Aktes $\left(\mathrm{K}^{1} / \mathrm{TS}^{3} / \mathrm{A}^{5}\right) \ldots \ldots \ldots \ldots 1$

Fragmentarische Fassung des ersten Aktes $\left(\mathrm{K}^{1} / \mathrm{TS}^{3} / \mathrm{A}^{6}\right) \ldots \ldots \ldots \ldots 7$

Fragmentarische Fassung des ersten Aktes $\left(\mathrm{K}^{1} / \mathrm{TS}^{3} / \mathrm{A}^{8}\right) \ldots \ldots \ldots 6$

Fassung des ersten Aktes $\left(\mathrm{K}^{1} / \mathrm{TS}^{3} / \mathrm{A}^{10}\right) \ldots \ldots \ldots \ldots$. . . . . . . . . . . . . . . . . . . . . . . .

Fragmentarische Fassung des ersten Bildes $\left(\mathrm{K}^{1} / \mathrm{TS}^{3} / \mathrm{A}^{12}\right) \ldots \ldots . \ldots$. . . . . 84

Konzeption 2: Ein Sklavenball - Toxilus . . . . . . . . . . . . . . . . 93

Fragmentarische Fassung des ersten Bildes $\left(\mathrm{K}^{2} / \mathrm{TS}^{1} / \mathrm{A}^{3}\right) \ldots \ldots . \ldots . \ldots 94$

Fragmentarische Fassung des ersten Bildes $\left(\mathrm{K}^{2} / \mathrm{TS}^{1} / \mathrm{A}^{4}\right) \ldots \ldots$. . . . . . . . . . . . . . . . . . . . . .

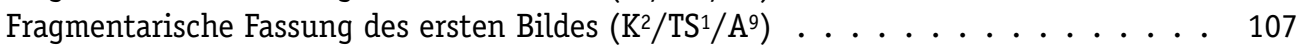

Strukturplan in drei Bildern $\left(\mathrm{K}^{2} / \mathrm{E}^{1}\right) \ldots \ldots \ldots$. . . . . . . . . . . . . . . . . . . . . . . . . . . . .

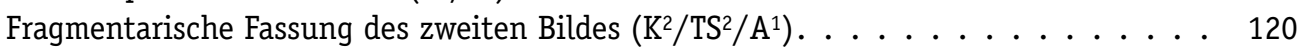

Fassung des zweiten Bildes $\left(\mathrm{K}^{2} / \mathrm{TS}^{2} / \mathrm{A}^{2}\right) \ldots \ldots \ldots \ldots \ldots$

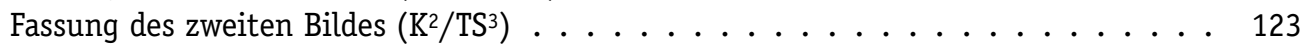

Konfigurationsplan $\left(\mathrm{K}^{2} / \mathrm{E}^{2}\right) \ldots \ldots \ldots \ldots \ldots \ldots$

Fragmentarische Fassung des dritten Bildes $\left(\mathrm{K}^{2} / \mathrm{TS}^{4}\right) \ldots \ldots \ldots 132$

Notiz $\left(\mathrm{K}^{2} / \mathrm{E}^{3}\right) \ldots \ldots \ldots \ldots \ldots \ldots$

Dialogskizze zum III. Bild $\left(\mathrm{K}^{2} / \mathrm{E}^{4}\right) \ldots \ldots \ldots \ldots \ldots$. . . . . . . . . . . 138

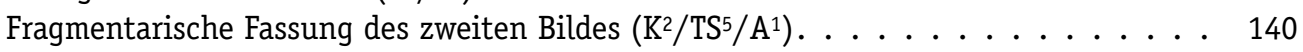

Fragmentarische Fassung des zweiten Bildes $\left(\mathrm{K}^{2} / \mathrm{TS}^{5} / \mathrm{A}^{3}\right) \ldots \ldots \ldots$. . . . . . . . . . . . . . . . . . . . . .

Fragmentarische Fassung des zweiten Bildes $\left(\mathrm{K}^{2} / \mathrm{TS}^{5} / \mathrm{A}^{7}\right) \ldots \ldots$. . . . . . . . . . . 147

Fragmentarische Fassung des zweiten Bildes $\left(\mathrm{K}^{2} / \mathrm{TS}^{5} / \mathrm{A}^{11}\right) \ldots \ldots$. . . . . . . . 156

Konzeption 3: Ein Sklavenball mit Gesang und Tanz in drei Akten . . . . . . . . . . . . . 163

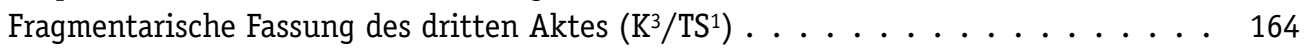

Replik, Dialogskizze $\left(\mathrm{K}^{3} / \mathrm{E}^{1}-\mathrm{E}^{2}\right) \ldots \ldots \ldots \ldots$. . . . . . . . . . . . . . . . . . . . 168

Konfigurationsplan, Dialogskizze $\left(\mathrm{K}^{3} / \mathrm{E}^{3}-\mathrm{E}^{4}\right) \ldots \ldots \ldots$. . . . . . . . . . . . . . . . . . . . . . . . .

Fassung des zweiten Aktes $\left(\mathrm{K}^{3} / \mathrm{TS}^{2} / \mathrm{A}^{1}\right) \ldots \ldots \ldots \ldots \ldots$. . . . . . . . . . . . . . . . . . . . . . . . . . . . . . . .

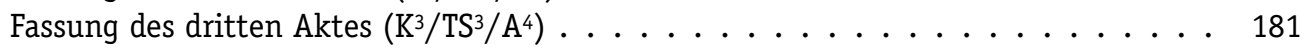

Fragmentarische Fassung $\left(\mathrm{K}^{3} / \mathrm{TS}^{4}\right) \ldots \ldots \ldots \ldots$

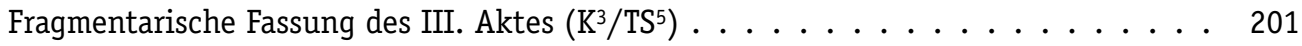

Fragmentarische Fassung $\left(\mathrm{K}^{3} / \mathrm{TS}^{6}\right) \ldots \ldots \ldots 202$

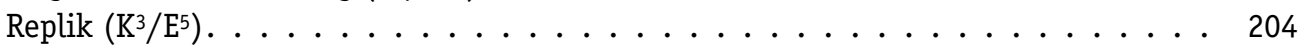

Dialogskizze $\left(\mathrm{K}^{3} / \mathrm{E}^{6}\right) \ldots \ldots \ldots \ldots \ldots$

Fragmentarische Fassung des dritten Aktes $\left(\mathrm{K}^{3} / \mathrm{TS}^{7} / \mathrm{A}^{2}\right) \ldots \ldots$. . . . . . . . . . . . . . . . . . . . 208

Fragmentarische Fassung des dritten Aktes $\left(\mathrm{K}^{3} / \mathrm{TS}^{7} / \mathrm{A}^{5}\right) \ldots \ldots . . \ldots . . \ldots 212$

Fragmentarische Fassung des dritten Aktes $\left(\mathrm{K}^{3} / \mathrm{TS}^{7} / \mathrm{A}^{6}\right) \ldots \ldots . . \ldots . . \ldots 221$

Fragmentarische Fassung des dritten Aktes $\left(\mathrm{K}^{3} / \mathrm{TS}^{7} / \mathrm{A}^{8}\right) \ldots \ldots . \ldots . \ldots . \ldots 227$ 
Fassung des dritten Aktes $\left(\mathrm{K}^{3} / \mathrm{TS}^{7} / \mathrm{A}^{11}\right) \ldots \ldots \ldots 233$

Fassung des ersten Aktes $\left(\mathrm{K}^{3} / \mathrm{TS}^{8} / \mathrm{A}^{3}\right) \ldots \ldots \ldots \ldots$

Fragmentarische Fassung des dritten Aktes $\left(\mathrm{K}^{3} / \mathrm{TS}^{7} / \mathrm{A}^{18}\right) \ldots \ldots . \ldots 265$

Gesamtfassung Ein Sklavenball $\left(\mathrm{K}^{3} / \mathrm{TS}^{9}\right)$. . . . . . . . . . . . . . . . 282

Konzeption 4: Ein Sklavenball mit Gesang und Tanz in drei Akten - Masken . . . . . . . 319

Fassung des ersten Aktes $\left(\mathrm{K}^{4} / \mathrm{TS}^{1} / \mathrm{A}^{2}\right) \ldots \ldots \ldots . \ldots \ldots$

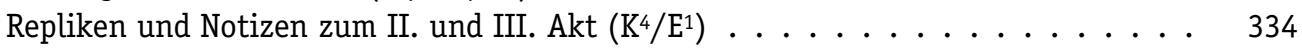

Endfassung Ein Sklavenball $\left(\mathrm{K}^{4} / \mathrm{TS}^{2} / \mathrm{A}^{3}\right) \ldots \ldots \ldots$

\section{Band 2}

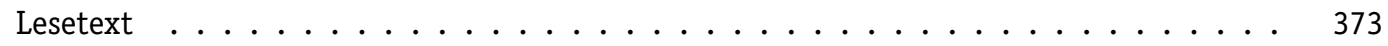

Konzeption 5: Ein Sklavenball / Pompeji - Adaptierungsarbeiten . . . . . . . . . . 375

Strukturpläne $\left(\mathrm{K}^{5} / \mathrm{E}^{1}-\mathrm{E}^{4}\right) \ldots \ldots \ldots \ldots \ldots \ldots$

Strukturplan in sieben Bildern $\left(\mathrm{K}^{5} / \mathrm{E}^{5}\right) \ldots \ldots \ldots$

Fassung des zweiten Bildes $\left(\mathrm{K}^{5} / \mathrm{TS}^{1}\right) \ldots \ldots \ldots$. . . . . . . . . . . . . . . . . . . . . . . . . . . . . . . . .

Dialogskizze zum vierten Bild $\left(\mathrm{K}^{5} / \mathrm{E}^{6}\right) \ldots \ldots \ldots$. . . . . . . . . . . . . . . . . . . . . . . 388

Fassung des zweiten Bildes $\left(\mathrm{K}^{5} / \mathrm{TS}^{2}\right) \ldots \ldots \ldots \ldots \ldots \ldots$

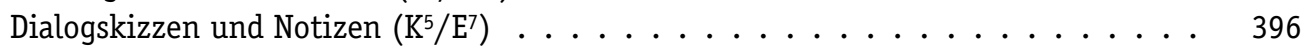

Fragmentarische Fassung des 5. Bildes $\left(\mathrm{K}^{5} / \mathrm{TS}^{3}\right) \ldots \ldots \ldots \ldots$

Fassung des ersten Aktes $\left(\mathrm{K}^{5} / \mathrm{TS}^{4}\right) \ldots \ldots \ldots . \ldots \ldots$

Fragmentarische Fassung $\left(\mathrm{K}^{5} / \mathrm{TS}^{5} / \mathrm{A}^{2}\right) \ldots \ldots \ldots \ldots$

Dialogskizze $\left(\mathrm{K}^{5} / \mathrm{E}^{8}\right) \ldots \ldots \ldots \ldots$. . . . . . . . . . . . . . 410

Fragmentarische Fassung des zweiten Bildes („Am nächsten Tage“) $\left(\mathrm{K}^{5} / \mathrm{TS}^{6} / \mathrm{A}^{1}\right)$. . . 412

Fragmentarische Fassung des zweiten Bildes ("Am nächsten Tage“) $\left(\mathrm{K}^{5} / \mathrm{TS}^{6} / \mathrm{A}^{4}\right)$. . . 415

Fassung des zweiten Bildes ("Am nächsten Tage $\left.{ }^{\prime \prime}\right)\left(\mathrm{K}^{5} / \mathrm{TS}^{6} / \mathrm{A}^{8}\right) \ldots \ldots 418$

Fragmentarische Fassung $\left(\mathrm{K}^{5} / \mathrm{TS}^{7}\right) \ldots \ldots \ldots$. . . . . . . . . . . . . . . . . . . . . . . . . . . . . . . . 426

Fassung des V. Bildes $\left(\mathrm{K}^{5} / \mathrm{TS}^{8}\right) \ldots \ldots \ldots \ldots \ldots \ldots$. . . . . . . . . . . . . . . . . . . . . . . . . . . . 437

Dialogskizze zum VI. Bild $\left(\mathrm{K}^{5} / \mathrm{E}^{9}\right) \ldots \ldots \ldots \ldots$. . . . . . . . . . . . . . . . . . . . . . . . . . . . . . . . . . .

Dialogskizze zum sechsten Bild $\left(\mathrm{K}^{5} / \mathrm{E}^{10}\right) \ldots \ldots \ldots$. . . . . . . . . . . . . . . . . . . . . . . . . . . . . .

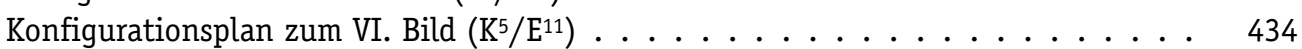

Fragmentarische Fassung des VII. Bildes $\left(\mathrm{K}^{5} / \mathrm{TS}^{9}\right) \ldots \ldots \ldots$. . . . . . . . . . . . . . . . . . . . . . 436

Strukturpläne in vier Bildern $\left(\mathrm{K}^{5} / \mathrm{E}^{12}-\mathrm{E}^{13}\right) \ldots \ldots \ldots$. . . . . . . . . . . . . . . . . . . . . . 438

Fassung des zweiten Bildes ("Am nächsten Tage $\left.{ }^{\prime \prime}\right)\left(\mathrm{K}^{5} / \mathrm{TS}^{6} / \mathrm{A}^{16}\right) \ldots . . . . . . . .440$

Fassung des dritten Bildes ("Wieder vor der Villa“) $\left(\mathrm{K}^{5} / \mathrm{TS}^{10}\right) \ldots \ldots$. . . . . . . . . . . . . . . . . . . . . . 45

Dialogskizze, Strukturplan in acht Bildern $\left(\mathrm{K}^{5} / \mathrm{E}^{14}-\mathrm{E}^{15}\right) \ldots \ldots . \ldots . \ldots 42$

Konzeption 6: Pompeji. Komödie in drei Teilen (neun Bildern) . . . . . . . . . . . 455

Strukturpläne in neun und vier Bildern, Notiz $\left(\mathrm{K}^{6} / \mathrm{E}^{1}-\mathrm{E}^{3}\right) \ldots \ldots . . \ldots 456$

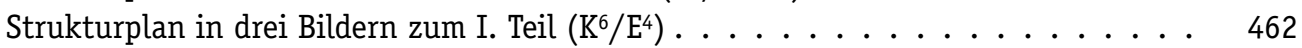

Fragmentarische Fassung des zweiten Bildes ("Am nächsten Tage“) $\left(\mathrm{K}^{6} / \mathrm{TS}^{1} / \mathrm{A}^{3}\right)$. . . 464

Dialogskizze und Repliken $\left(\mathrm{K}^{6} / \mathrm{E}^{5}\right) \ldots \ldots \ldots$. . . . . . . . . . . 468

Fragmentarische Fassung des zweiten Bildes ("Am nächsten Tage“) $\left(\mathrm{K}^{6} / \mathrm{TS}^{1} / \mathrm{A}^{6}\right)$. . . 470

Fragmentarische Fassung $\left(\mathrm{K}^{6} / \mathrm{TS}^{2}\right) \ldots \ldots \ldots \ldots$. . . . . . . . . . . . . . . . . . . . . . . . . . . . .

Fragmentarische Fassung $\left(\mathrm{K}^{6} / \mathrm{TS}^{3}\right) \ldots \ldots \ldots$. . . . . . . . . . . . . . 480

Fragmentarische Fassung des vierten Bildes ("Bei Bagnio“) $\left(\mathrm{K}^{6} / \mathrm{TS}^{4}\right)$. . . . . . . . 483

Fragmentarische Fassung des dritten Bildes (,Wieder vor der Villa“) $\left(\mathrm{K}^{6} / \mathrm{TS}^{5}\right)$. . . . 485

Repliken zum 2. Bild $\left(\mathrm{K}^{6} / \mathrm{E}^{6}\right) \ldots \ldots . \ldots . \ldots . \ldots 490$

Fassung des zweiten Bildes („Wieder vor der Villa“) $\left(\mathrm{K}^{6} / \mathrm{TS}^{8} / \mathrm{A}^{3}\right) \ldots \ldots$. . . . . . . 492

Fassung des dritten Bildes ("In einem Keller") $\left(\mathrm{K}^{6} / \mathrm{TS}^{9}\right)$. . . . . . . . . . . . . . . . . 497

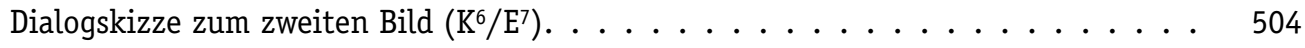


Replik und Dialogskizze $\left(\mathrm{K}^{6} / \mathrm{E}^{8}\right) \ldots \ldots \ldots \ldots 06$

Strukturpläne $\left(\mathrm{K}^{6} / \mathrm{E}^{9}-\mathrm{E}^{10}\right) \ldots \ldots \ldots \ldots \ldots$

Fassung des dritten Bildes ("In einem Keller") $\left(\mathrm{K}^{6} / \mathrm{TS}^{10}\right)$. . . . . . . . . . . . . . 510

Fragmentarische Fassung des dritten Bildes („In einem Keller $\left.{ }^{\prime \prime}\right)\left(\mathrm{K}^{6} / \mathrm{TS}^{11} / \mathrm{A}^{1}\right)$. . . . 518

Fassung des IV. Bildes ("Auf dem Sklavenmarkt") $\left(\mathrm{K}^{6} / \mathrm{TS}^{12}\right)$. . . . . . . . . . . . . . 519

Fragmentarische Fassung des vierten Bildes („Beim Sklavenhändler“) $\left(\mathrm{K}^{6} / \mathrm{TS}^{13} / \mathrm{A}^{1}\right)$. . 521

Fassung des vierten Bildes („Beim Sklavenhändler“) $\left(\mathrm{K}^{6} / \mathrm{TS}^{13} / \mathrm{A}^{2}\right)$. . . . . . . . . . 524

Dialogskizze, Replik $\left(\mathrm{K}^{6} / \mathrm{E}^{11}-\mathrm{E}^{12}\right) \ldots \ldots \ldots \ldots$. . . . . . . . . . . . . . . . . . . . . . . 532

Fassung des sechsten Bildes ("Im Zirkus") $\left(\mathrm{K}^{6} / \mathrm{TS}^{15}\right)$. . . . . . . . . . . . . 534

Fragmentarische Fassung des siebenten Bildes $\left(\mathrm{K}^{6} / \mathrm{TS}^{16}\right)$. . . . . . . . . . . . 540

Konzeption 7: Pompeji. Komödie eines Erdbebens in sechs Bildern . . . . . . . . . . . . 541

Fragmentarische Fassung des VI. Bildes $\left(\mathrm{K}^{7} / \mathrm{TS}^{1}\right) \ldots \ldots \ldots . \ldots \ldots 42$

Fragmentarische Fassung des sechsten Bildes ("Im Zirkus“) $\left(\mathrm{K}^{7} / \mathrm{TS}^{2} / \mathrm{A}^{1}\right)$. . . . . . . . 544

Fragmentarische Fassung des sechsten Bildes („Im Zirkus“) $\left(\mathrm{K}^{7} / \mathrm{TS}^{2} / \mathrm{A}^{3}\right)$. . . . . . . . 546

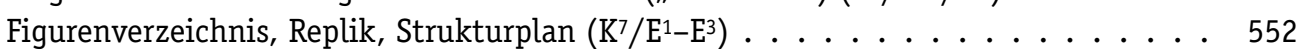

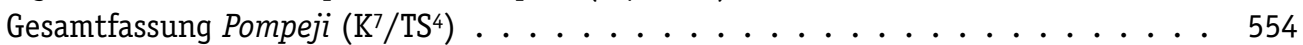

Endfassung Pompeji $\left(\mathrm{K}^{7} / \mathrm{TS}^{5}\right) \ldots \ldots \ldots \ldots \ldots \ldots$

Werkverzeichnis $\left(\mathrm{K}^{7} / \mathrm{E}^{4}\right) \ldots \ldots \ldots \ldots$. . . . . . . . . . . . . 634

Ein Sklavenball mit Gesang und Tanz in drei Akten

(Endfassung, emendiert). . . . . . . . . . . . . . 637

Pompeji. Komödie eines Erdbebens in sechs Bildern

(Endfassung, emendiert). . . . . . . . . . . . . . . . . 671

Kommentar . . . . . . . . . . . . . . . . . . 707

Chronologisches Verzeichnis . . . . . . . . . . . . . . . 709

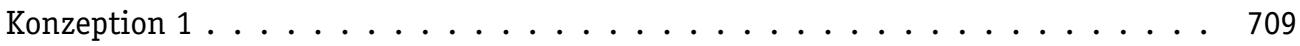

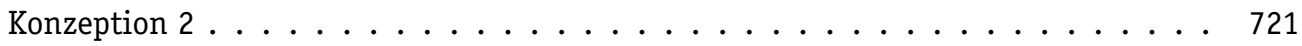

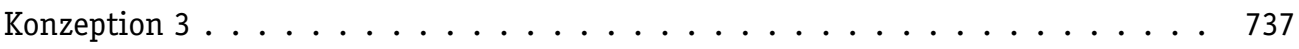

Konzeption $4 \ldots \ldots \ldots \ldots 757$

Konzeption $5 \ldots \ldots \ldots \ldots 762$

Konzeption $6 \ldots \ldots \ldots \ldots$

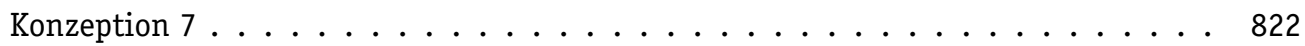

Endfassungen, emendiert . . . . . . . . . . . . . . 830

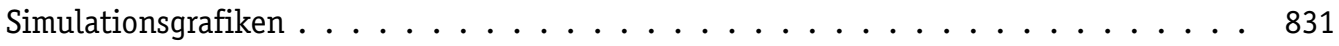

$\mathrm{K}^{1} / \mathrm{TS}^{3} / \mathrm{A}^{1}-\mathrm{A}^{13} \ldots \ldots \ldots \ldots \ldots \ldots \ldots \ldots$

$\mathrm{K}^{2} / \mathrm{TS}^{1} / \mathrm{A}^{1}-\mathrm{A}^{11} \ldots \ldots \ldots \ldots \ldots \ldots \ldots$

$\mathrm{K}^{2} / \mathrm{TS}^{5} / \mathrm{A}^{1}-\mathrm{A}^{14} \ldots \ldots \ldots \ldots \ldots \ldots$

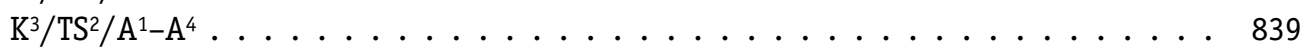

$\mathrm{K}^{3} / \mathrm{TS}^{7} / \mathrm{A}^{1}-\mathrm{A}^{11} \ldots \ldots \ldots \ldots \ldots \ldots \ldots \ldots \ldots \ldots$

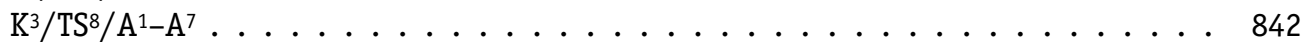

$\mathrm{K}^{3} / \mathrm{TS}^{7} / \mathrm{A}^{12}-\mathrm{A}^{21} \ldots \ldots \ldots \ldots \ldots \ldots \ldots \ldots \ldots \ldots \ldots$

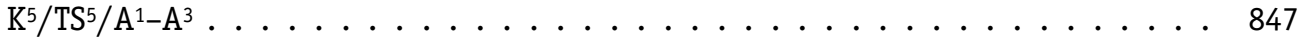

$\mathrm{K}^{5} / \mathrm{TS}^{6} / \mathrm{A}^{1}-\mathrm{A}^{16} \ldots \ldots \ldots \ldots \ldots \ldots \ldots \ldots$

$\mathrm{K}^{6} / \mathrm{TS}^{1} / \mathrm{A}^{1}-\mathrm{A}^{10} \ldots \ldots \ldots \ldots \ldots \ldots \ldots \ldots$

$\mathrm{K}^{6} / \mathrm{TS}^{8} / \mathrm{A}^{1}-\mathrm{A}^{5} \ldots \ldots \ldots \ldots \ldots \ldots \ldots$

$\mathrm{K}^{6} / \mathrm{TS}^{11} / \mathrm{A}^{1}-\mathrm{A}^{11} \ldots \ldots \ldots \ldots \ldots \ldots \ldots$

$\mathrm{K}^{6} / \mathrm{TS}^{13} / \mathrm{A}^{1}-\mathrm{A}^{11} \ldots \ldots \ldots \ldots \ldots \ldots \ldots \ldots \ldots \ldots \ldots$

$\mathrm{K}^{7} / \mathrm{TS}^{2} / \mathrm{A}^{1}-\mathrm{A}^{7} \ldots \ldots \ldots \ldots \ldots \ldots \ldots \ldots$

Dossier: Plautus-Intertexte in Ein Sklavenball / Pompeji . . . . . . . . . . . . . . . . . 857 


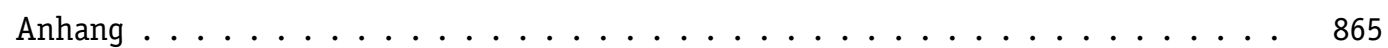

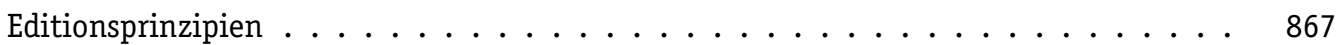

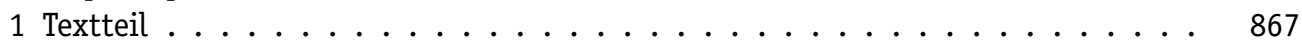

1.1 Genetisches Material . . . . . . . . . . . . . . . . . 867

1.1.1 Diplomatische Transkription und Faksimile (Entwürfe) . . . . . . . 867

1.1.2 Lineare Textkonstitutionen (Fassungen) . . . . . . . . . . . 868

1.1.3 Kritisch-genetischer Apparat. . . . . . . . . . . . . . 869

1.2 Emendierter Text (Endfassung) . . . . . . . . . . . . . . . . . 870

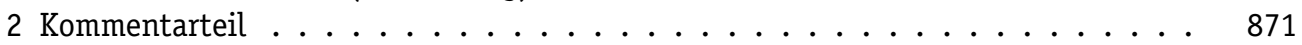

2.1 Chronologisches Verzeichnis . . . . . . . . . . . . . . . . 871

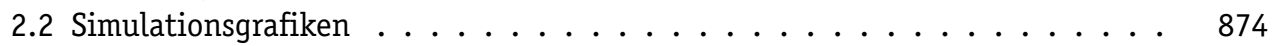

Siglen und Abkürzungen . . . . . . . . . . . . . . . 876

Literaturverzeichnis . . . . . . . . . . . . . . . . . . 879 